[original insight]

Diamond Open Access

\title{
Universal Knowledge Base
}

\author{
Open Mathematics Collaboration*†
}

August 4, 2020

\begin{abstract}
We present the STR simple rules to build a knowledge base with all scientific knowledge.
\end{abstract}

keywords: knowledge base, scientific knowledge, digital platform, artificial intelligence

The most updated version of this paper is available at https://osf.io/pwvzt/download

\section{Introduction}

0. Imagine all the scientific knowledge in a digital searchable platform [1].

\section{Simple rules to deploy a knowledge base}

1. $S=$ Insert each Scientific result with the minimum characters as possible.

2. $T=$ Tag it precisely.

3. $R=$ Review both $S$ and $T$ by specialists.

*All authors with their affiliations appear at the end of this paper.

†Corresponding author: mplobo@uft.edu.br | Join the Open Mathematics Collaboration 


\section{Examples}

4. Open Mathematics Knowledge Base [2]

5. The Self-Publishing Journal [3], specially if it publishes articles written in items [4], is a transitional solution toward the Universal Knowledge Base.

\section{Final Remarks}

6. $S+T=$ deployed by artificial intelligence

7. $\mathrm{R}=$ performed by human

\section{Open Invitation}

Review, add content, and co-author this paper $[4,5]$.

Join the Open Collaboration (https://bit.ly/ojmp-slack).

Send your contribution to mplobo@uft.edu.br.

\section{Open Science}

The latex file for this paper together with other supplementary files are available [6].

\section{Ethical conduct of research}

This original work was pre-registered under the OSF Preprints [7], please cite it accordingly [8]. This will ensure that researches are conducted with integrity and intellectual honesty at all times and by all means. 


\section{Acknowledgement}

+ Center for Open Science

https://www.cos.io

+ Open Science Framework

https://osf.io

\section{References}

[1] Nielsen, Michael. Reinventing discovery: the new era of networked science. Vol. 70. Princeton University Press, 2020.

[2] Lobo, Matheus P. "Open Mathematics Knowledge Base." OSF Preprints, 13 May 2020. https://doi.org/10.31219/osf.io/evq8a

[3] Lobo, Matheus P. "Self-publishing Journal." OSF Preprints, 17 June 2020. https://doi.org/10.31219/osf.io/782da

[4] Lobo, Matheus P. "Microarticles." OSF Preprints, 28 Oct. 2019. https://doi.org/10.31219/osf.io/ejrct

[5] Lobo, Matheus P. "Simple Guidelines for Authors: Open Journal of Mathematics and Physics." OSF Preprints, 15 Nov. 2019. https://doi.org/10.31219/osf.io/fk836

[6] Lobo, Matheus P. "Open Journal of Mathematics and Physics (OJMP)." OSF, 21 Apr. 2020. https://doi.org/10.17605/osf.io/6hzyp

[7] COS. Open Science Framework. https://osf.io

[8] Lobo, Matheus P. "Universal Knowledge Base." OSF Preprints, 21 June 2020. https://doi.org/10.31219/osf.io/pwvzt 


\section{The Open Collaboration}

Matheus Pereira Lobo (lead author, mplobo@uft.edu.br) $)^{1,2}$ https://orcid.org/0000-0003-4554-1372

${ }^{1}$ Federal University of Tocantins (Brazil)

${ }^{2}$ Universidade Aberta (UAb, Portugal) 